\title{
Primer Centro de Medicina del Viajero en un hospital público de la Provincia de Buenos Aires, República Argentina. Dos años de experiencia
}

\author{
Amadeo Esposto, María L. Yantorno, Karina Aguilera, Mónica Lares, Gloria de la Parra e Inés Roccia Rossi
}

\section{First Travel Medicine Center in a public hospital from Buenos Aires Province, Argentina Republic. The first 2 years of experience}

Introduction: Although Buenos Aires is the biggest province in Argentina, there was no program for Travel Medicine in any public hospital until 2008, when the Travel Medicine Center (CEMEVI) was established in our hospital. Objective: To analyze the first 24 months of experience in the CEMEVI. Results: A total of 278 travelers were assisted. Most of them consulted before traveling (pre-travel visits). The most common destinations were countries in South America and urban as well as rural areas. Travelling to malaria and yellow fever endemic countries represented $35 \%$ and $16 \%$ of the total of destinations, respectively. Only $4 \%$ were post-travel interviews. Conclusion: It is feasible and frutful to implement a Travel Medicine Center in the public health system.

Key words: Travel clinics, travellers, pre-travel consultation, travel advice.

Palabras clave: Clínicas de medicina del viajero, viajeros, consulta previaje, asesoramiento viajeros.

\section{Introducción}

A nualmente mil quinientos millones de personas viajan por vía aérea, y 700 millones de ellos cruzan barreras internacionales ${ }^{1}$. De acuerdo a lo informado por la Organización Mundial del Turismo, para el año 2010, las llegadas de turistas internacionales se estimaron en 940 millones $^{2}$.

El riesgo de enfermar durante un viaje está relacionado con el destino, el estado de salud del viajero, el propósito del viaje, la duración y la época del año, alojamiento, comportamiento y estilo de vida. Por tal motivo, los viajes de mochila y aventura, visitar a familiares y amigos, visitar zonas rurales y fuera de las rutas turísticas habituales, duración del viaje superior a cuatro semanas y expatriados son reconocidos factores de riesgo $0^{3,4,5,6}$.

Se estima que sobre 100.000 viajeros cuyos destinos sean a países en desarrollo y que permanezcan en ellos al menos un mes ${ }^{5}$ :

- 50.000 presentarán algún problema de salud.

- 8.000 necesitarán asistencia médica.

- 5.000 deberán guardar reposo en cama.

- 1.100 estarán incapacitados para trabajar.

- 300 necesitarán atención hospitalaria.

- 50 tendrán que ser evacuados de su destino de viaje.

- 1 fallecerá por la enfermedad adquirida durante el viaje.

Los traumas y las infecciones son las principales causas de enfermedad en los viajeros ${ }^{7}$. La historia de la medicina está jalonada de hechos vinculados a los desplazamientos de personas.

En estas circunstancias, se presentan innumerables condiciones que favorecen la aparición y diseminación de enfermedades. Son bien conocidos los brotes masivos de cólera, disentería, sarampión y otras afecciones en campos de refugiados ${ }^{8,9}$.

Marcadores moleculares hicieron posible reconocer que la cepa epidémica de Neisseria meningitidis serogrupo A en peregrinos a La Meca, provenía del sudeste asiático en el brote ocurrido en $1987^{9}$.

En recientes años han ocurrido nuevos brotes relacionados a viajeros.

En marzo de 2003 se alerta sobre la aparición de una enfermedad respiratoria aguda grave que comienza en la provincia de Guangdong (China) denominada posteriormente SARS (Severe Acute Respiratory Syndrome). Los viajeros internacionales fueron el medio a través de los cuales se produjo la rápida diseminación mundial y en mayo del 2003, esta enfermedad ya había afectado 32 países $^{10}$.

En el año 2009 viajeros internacionales que partían de México fueron los encargados de difundir la nueva cepa pandémica de influenza A H1N1 en el resto del mundo ${ }^{11}$.

En agosto de 2010 se identificaron en nuestro país dos casos importados de sarampión de personas que habían viajado al Mundial de fútbol en Sudáfrica, con nuevos casos posteriores en contactos ${ }^{12}$.

Por este motivo es que, a lo largo de los años, se ha incrementado en todo el mundo la práctica de la Medicina
Hospital Interzonal General de Agudos "General San Martín". Ciudad de La Plata. Provincia de Buenos Aires. República Argentina. Servicio de Infectología.

Los autores declaran no tener conflictos de interés.

Financiamiento: No hubo.

Recibido: 31 de marzo de 2011 Aceptado: 22 de marzo de 2012

Correspondencia a: María Laura Yantorno. mlaurayan@hotmail.com 
del Viajero, y de clínicas dedicadas al asesoramiento de los mismos antes, durante y después del viaje. A pesar de esto, su influencia en la Argentina ha sido limitada y acotada a unos pocos centros públicos y privados, la mayoría localizados en la Ciudad Autónoma de Buenos Aires (ciudad Capital de la República Argentina).

La Provincia de Buenos Aires, aun siendo la mayor de nuestro país, no contaba con ningún sitio para asesoramiento del viajero instalado en un hospital público hasta que en el año 2008 se crea el Centro de Medicina del Viajero (CEMEVI) en nuestro hospital utilizando la capacidad instalada disponible en el Servicio de Infectología y con personal médico entrenado a tal fin.

El objetivo de este trabajo es analizar de manera retrospectiva los primeros 24 meses de experiencia en el CEMEVI.

\section{Material y Métodos}

Se realizó un estudio retrospectivo, transversal, de los viajeros que consultaron al Centro durante los primeros dos años de funcionamiento (marzo de 2008-marzo de 2010). El CEMEVI forma parte del Servicio de Infectología del Hospital General San Martín de La Plata, un hospital universitario de aproximadamente 350 camas.

Al momento de la consulta se le entregó a cada viajero un cuestionario donde debieron completar con datos filiatorios y antecedentes personales, propósito del viaje, destino, duración, estilo de viaje, traslado a zonas de riesgo para fiebre amarilla y malaria e historia de vacunaciones previas. Se diseñó una base de datos la que fue analizada en el programa Excel, versión 2003.

Se analizaron también los viajeros que consultaron al regreso de su viaje y los motivos de consulta más frecuentes.

Se excluyeron las consultas realizadas por correo electrónico o vía telefónica.

\section{Resultados}

Durante el período de estudio se realizaron 278 consultas, de las cuales 267 viajeros (96\%) lo hicieron antes del viaje y 11 (4\%) luego del mismo (10 de ellos sin consulta pre-viaje), por lo cual se analizaron por separado.

Los viajeros con consulta pre-viaje presentaron las siguientes características: la edad media fue 37 años (6-81 años) y 45\% fueron de sexo masculino (121/267). Diecinueve viajeros $(7,1 \%)$ fueron inmunocomprometidos (portadores de VIH, neoplasias y uso de $\geq 10 \mathrm{mg}$ / día de prednisona o equivalente). No hubo consultas de mujeres embarazadas, y 7,8\% (21/267) de las consultas fueron personas menores a 15 años de edad. Ninguno de los viajeros fue derivado desde agencias de viajes.
Tabla 1. Características generales de los viajeros durante la evaluación en el previaje (n: 267)

\begin{tabular}{lcc|} 
Edad media & 37 años & $(6-81)$ \\
Sexo masculino & 121 & $(45,3)$ \\
Inmunocomprometidos & 19 & $(7,1)$ \\
Niños & 21 & $(7,8)$ \\
Días de consulta previo al viaje & 25,5 días & $(1-210)$ \\
Motivos del viaje & & \\
$\quad$ Vacaciones & 213 & $(80 \%)$ \\
Misioneros & 9 & $(3,3 \%)$ \\
$\quad$ Negocios & 8 & $(3 \%)$ \\
Estudios & 8 & $(3 \%)$ \\
Otros & 29 & $(10,8 \%)$ \\
Tipo de viaje & & $(61,4 \%)$ \\
Urbano-rural & 164 & $(35,2 \%)$ \\
Urbano & 94 & $(3,4 \%)$ \\
Rural & 9 & $(1,4 \%)$ \\
Alojamiento & $41,8 \%)$ \\
Hoteles u hostel & & \\
Casa de familia & 41 & $(15,3 \%)$ \\
Carpa & &
\end{tabular}

El promedio de días de consulta previo al viaje fue de 25,5 días (rango 1- 210 días), con una mediana de 30 días. El 71\% de los viajeros (n: 190) tuvo una estadía promedio menor a cuatro semanas.

En $80 \%$ de los casos los viajes fueron por vacaciones $(213 / 267)$, en $3,3 \%$ misioneros (9/267), en tanto negocios y estudios 3\% (8/267) en cada caso En el resto, el motivo de viaje se debió a causas tales como adopción, asistir a congresos, y visitar familiares y amigos. En cuanto al tipo de viaje, la mayoría fue a zona urbano-rural $(61,4 \%)$, mientras que $35,2 \%$ eligió zona urbana; sólo 9 viajeros visitaron zonas rurales exclusivamente. En 12\% (32/267) de los casos fueron viajes de mochila y aventura.

El tipo de alojamiento también fue variable. El 52,8\% $(141 / 267)$ de los viajeros eligió alojarse en hoteles $u$ hosteles, mientras que $15,3 \%(41 / 267)$ prefirió hacerlo en casas de familia; sólo 4 (1,4\%) utilizaron carpas exclusivamente. El resto utilizó diferentes tipos de alojamiento durante el viaje (Tabla 1).

Pese a que en algunos casos no pudo establecerse un itinerario totalmente preciso, los destinos elegidos, en orden de frecuencia fueron: a países de América del Sur en 178/267 (66\%), Asia en 39/267 (14,6\%), África en $35 / 267(13,1 \%)$, América Central y Caribe en 11/267 (4,1\%), y el resto a Europa e Islas del Pacífico (Figura 1).

Los países más visitados en América del Sur fueron: Perú 53/178 (29,7\%), Brasil 47/178 (26,4\%), Bolivia 45/178 (25,2\%) y Argentina 35/178 (19,6\%). El destino asiático preferido fue la India 19/39 (48,7\%). 
El 35\% de los viajeros (94/267) eligió destinos con riesgo para adquirir malaria y 16\% (43/267) lo hizo a países con riesgo de fiebre amarilla.

Once viajeros consultaron al regreso de su viaje, de los cuales sólo uno recibió asesoramiento pre-viaje. La mayoría de estos viajeros eran de sexo masculino (72\%) y una edad promedio de 33 años (24-51). El destino elegido fue a países de América del Sur en $72,7 \%$ de los casos, siendo Brasil el principal país visitado (5/11). Cuatro viajeros $(36 \%)$ consultaron por fiebre $>38^{\circ} \mathrm{C}$, otros cuatro $(36 \%)$ por presentar lesiones en piel, en tanto que por diarrea, cefalea y síndrome meníngeo lo hicieron un viajero en cada caso.

En los pacientes febriles, se realizaron los estudios considerados pertinentes según el cuadro clínico que motivó la consulta. Entre estos se incluyeron: química general, hemocultivos, coprocultivo y copro-parasitológico seriado, gota gruesa para malaria, serologías de dengue, fiebre tifoidea, VIH, hepatitis A, B y C. En dos de estos pacientes (2/4) se diagnosticó dengue serotipo 2 (serología IgM positiva y posteriormente seroconversión). Ambos pacientes habían viajado a la ciudad de Río de Janeiro (Brasil). En el tercer paciente, que había viajado a Asia, se diagnosticó fiebre tifoidea por serología y en el cuarto paciente que había viajado a diversos países del Cono Sur, todos los resultados solicitados fueron negativos.

En cuanto a los viajeros que presentaron lesiones en piel, los diagnósticos fueron larva migrans cutánea, urticaria gigante en dos casos, y picadura de insectos.

Un sólo paciente fue hospitalizado por presentar un síndrome meníngeo luego de viajar a Paraguay. En este caso se realizó punción lumbar para cultivo de microorganismos comunes, tinción con tinta china, RPC para herpesvirus y serología para flavivirus siendo la IgM positiva para fiebre amarilla tanto en el líquido céfaloraquídeo como en sangre.

Todos los viajeros tuvieron buena evolución clínica.

\section{Discusión}

La práctica de la Medicina del Viajero se ha desarrollado notablemente en los últimos 25 años. La Infectious Diseases Society of America (IDSA) ha identificado cuatro factores primordiales para que ello acontezca: primero, el incremento incesante en el número de viajeros internacionales, con la diversidad y complejidad de sus itinerarios y actividades. Segundo, estudios epidemiológicos que han definido el riesgo de adquirir infecciones durante un viaje, en especial malaria y diarrea. Tercero, el desarrollo farmacéutico en especial en el área de vacunas y por último, la necesidad de ampliar la asesoría no sólo a la prevención y profilaxis de las enfermedades infecciosas sino incluir temas tales como el mal de altura,

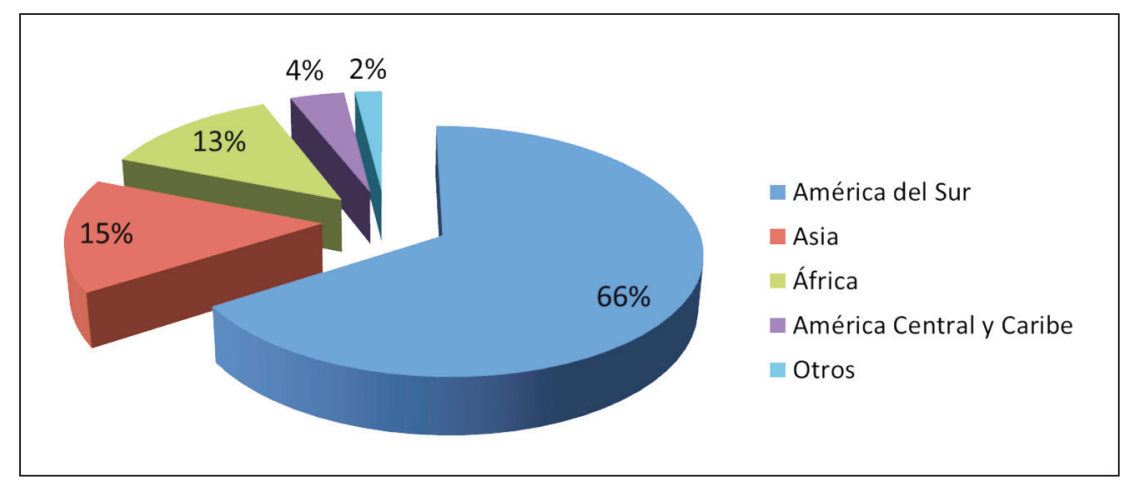

Figura 1. Destinos más frecuentes elegidos por los viajeros.

comportamientos, estilos de vida, seguridad, accidentes y acceso a un sistema de salud si fuera necesario ${ }^{13}$.

En nuestro Centro se ofrece un asesoramiento pre, intra y post viaje. En la consulta previa se realiza una evaluación de riesgo en base a su historia personal e itinerario y características de viaje y se realizan las recomendaciones en cuanto a vacunas, pautas generales y medicamentos para minimizar los riesgos de enfermedades durante el mismo. En nuestra experiencia la mediana de días de consulta previa al viaje fue de 30 días, coincidiendo con las recomendaciones habituales (4 a 6 semanas de anticipación $)^{3,6}$. La mayoría de los viajes se realizaron con motivo de vacaciones ( $80 \%$ ) en tanto que visitar familiares $\mathrm{y}$ amigos fue motivo de pocas consultas.

En la literatura médica, los motivos más frecuentes de viaje son vacaciones $(50 \%)$, visitar familiares y amigos $(26 \%)$, negocios $(16 \%)$, y otros motivos ${ }^{2}$. Aproximadamente $8 \%$ de los viajeros que visitan países en desarrollo requieren atención médica en su destino o al regreso ${ }^{14}$. En nuestro caso, pese a que a cada viajero se les brindó un contacto vía correo electrónico mientras estaban en viaje, no recibimos consultas, y la mayoría de los viajeros que consultaron al regreso no habían hecho consulta previa en nuestro Centro.

Si bien muchas de estas enfermedades son autolimitadas tales como diarrea, infecciones respiratorias y enfermedades cutáneas, otras como la malaria, dengue o fiebre amarilla tienen gran importancia personal y epidemiológica con elevada morbi-mortalidad y posibilidad de transmisión territorial. Sin embargo, son los accidentes la principal causa de muerte y discapacidad prevenibles en el viajero. El costo de atención de todas estas eventualidades es enorme.

Tradicionalmente, el asesoramiento al viajero era brindado por agentes de viaje ${ }^{15}$ o médicos de familia; sin embargo, la evidencia demostró que no siempre ésta era adecuada y consistente ${ }^{1}$. En un estudio realizado por Hatz y cols., sólo $11 \%$ de los médicos suizos y $1 \%$ de los alemanes brindaron recomendaciones adecuadas 
para la protección de malaria y vacunas ${ }^{16}$. Keystone y cols. demostraron que la asesoria pre-viaje brindada por clínicas de Estados Unidos y Canadá fue muy variable, y que en 20 a $75 \%$ de los asesoramientos las vacunas recomendadas fueron incorrectas (indicación inadecuada de vacuna para fiebre amarilla, falta de refuerzos contra tétanos y polio, etc. $)^{17}$.

Esto refleja la compleja y multidisciplinaria actividad de la especialidad y de la imperiosa necesidad de formar recursos humanos idóneos aumentando los centros de asesoramiento para facilitar el acceso de la población.

Con la re-emergencia de la fiebre amarilla en Brasil se crea el CEMEVI, que, en sus inicios, estuvo a cargo de una médica infectóloga (MLY) cuya formación la realizara en el Instituto de Medicina Tropical Alexander von Humboldt del Hospital Cayetano Heredia de la ciudad de Lima (Perú), en el Centro Municipal de Patologías Regionales Argentina y Medicina Tropical (CEMPRA-MT) ubicado en el Hospital F.J. Muñiz de la Ciudad Autónoma de Buenos Aires (Argentina) y los cursos de Medicina del Viajero dictados por la Sociedad Latinoamericana de Medicina del Viajero (SLAMVI). Actualmente es atendido, además, por otros profesionales especializados en enfermedades infecciosas que han recibido similar adiestramiento.

Las guías de consejería y avisos de alerta por brotes utilizados por el CEMEVI provienen de variadas fuentes: CDC Travelers' Health, International Society of Travel Medicine (ISTM), OMS/OPS, Sociedad Latinoamericana de Medicina del Viajero, Sociedad Argentina de Infectología, Sistema de Vigilancia Epidemiológica de Córdoba (SIVECOR) y el Boletín Epidemiológico del Ministerio de Salud de la Nación Argentina, entre las más importantes.

El CEMPRA-MT es un renombrado Centro argentino de referencia de la especialidad, que desarrolla esta actividad desde la década de 1980. Es de jurisdicción municipal y, hasta la creación de nuestro centro (jurisdicción provincial), era el único funcionando en un hospital público en el país.

La explicación quizás radique en las peculiaridades de nuestro sistema de salud compuesto de tres subsectores: el público (jurisdicción nacional, provincial y municipal), el privado y el de la seguridad social (Obras Sociales) lo que permite una amplia cobertura poblacional. Sin embargo, la respuesta en muchas oportunidades es segmentada y heterogénea, siendo evidente que la asesoría del viajero no ha estado prioritariamente en la agenda de salud del país ya que se encontraba limitada y acotada a unos pocos sitios encargados de tal actividad, tanto en el ámbito de la medicina privada como pública y la mayoría ubicados en la Ciudad Autónoma de Buenos Aires.

En la experiencia recogida en estos primeros dos años hemos observado que la mayoría de las consultas fueron de personas jóvenes e inmunocompetentes y no hemos recibido consultas de mujeres embarazadas. Los países de América del Sur fueron los destinos elegidos en su mayoría, y esto concuerda con lo informado por la Organización Mundial del Turismo en el año 2011, donde la llegada de turistas internacionales creció aproximadamente $5 \%$ con respecto al año anterior, y que América del Sur tuvo un crecimiento notablemente mayor que la media mundial siendo aproximadamente de $15 \%{ }^{2}$.

Además, es de suponer también que la cercanía, los menores costos y los amplios atractivos culturales y paisajísticos de esta zona hacen que los viajeros argentinos que nos consultaron eligieran visitar países vecinos tales como Bolivia, Perú y Brasil. Asimismo dentro de la escasa bibliografía latinoamericana al respecto, $86 \%$ de los viajeros chilenos eligieron también Sudamérica como destino ${ }^{18}$.

Desde que a principios del año 2008 se confirmaron cuatro epizootias en monos Alouatta caraya por fiebre amarilla en la provincia de Misiones (Argentina) y luego de la confirmación de nueve casos autóctonos en huma$\operatorname{nos}^{19}$, el Ministerio de Salud de la Nación recomienda la aplicación de la vacuna de fiebre amarilla para los viajeros a zonas de riesgo, incluidas las Cataratas del Iguazú, en la provincia de Misiones. Pese a esto, sólo 35 personas buscaron asesoramiento para viajar por nuestro país, incluido el norte argentino y el litoral. Probablemente se deba a falta de conocimiento por parte de la población de los riesgos que se pueden adquirir aún viajando dentro del mismo país; a falta de asesoramiento de las agencias de turismo y a una inadecuada difusión por parte de los organismos de salud.

Tal como acontece en todos los hospitales públicos argentinos, sólo se proporcionan las vacunas incluidas en el Calendario Nacional de Vacunación. En nuestra ciudad se dispone de un centro para vacunación de fiebre amarilla con la extensión del certificado con validez internacional.

Sólo once viajeros consultaron en el post-viaje, la mayoría por fiebre, alteraciones cutáneas y diarrea coincidiendo con los datos reportados en la literatura científica $^{20-23}$.

Quisiéramos destacar que ningún viajero consultó al CEMEVI por derivación desde una agencia de viajes a pesar de la amplia difusión periodística y de folletería que se hiciera, incluso en las empresas vinculadas al turismo de nuestra ciudad. Esto habla a las claras de la falta de conciencia empresarial para ofrecer un servicio gratuito de prevención de enfermedades a sus clientes.

Si bien entendemos que el presente trabajo tiene limitaciones, dadas fundamentalmente por el escaso número de eventos analizados, su carácter retrospectivo y proveniente de un solo centro, creemos que tiene el interés de dar a conocer datos locales (sumamente escasos en la literatura médica) y de poner en evidencia que es posible 
montar un centro de estas características en el subsector público de salud, absolutamente gratuito con el recurso existente (edilicio, informático, humano), sin presupuestos adicionales, sólo basado en el interés profesional, con el adiestramiento adecuado y el apoyo de las autoridades de las instituciones sanitarias.

Esto permitió encarar en nuestro medio una actividad insólitamente relegada en la República Argentina.

Agradecimientos: al Profesor Dr. Eduardo Gotuzzo del Instituto de Medicina Tropical "Alexander von Humboldt" de la Universidad Peruana Cayetano Heredia (Lima, Perú) y a los doctores Tomas Orduna (Jefe de Servicio), Susana LLoveras, Sofía Echazarreta y equipo del CEMPRA-MT (Ciudad Autónoma de Buenos Aires, Argentina) por su permanente estímulo y apoyo.

\section{Resumen}

Introducción: La Provincia de Buenos Aires, a pesar de ser la mayor de nuestro país, no contaba con ningún sitio de asesoramiento al viajero instalado en un hospital público, hasta que en el año 2008 se crea el Centro de Medicina del Viajero (CEMEVI) en nuestro hospital. Objetivo: analizar de manera retrospectiva los primeros 24 meses de experiencia en el CEMEVI. Resultados: Recibimos un total de 278 consultas, la mayoría durante el pre-viaje, a países de América del Sur, a sitios urbanos- rurales, de los cuales 35 y $16 \%$ de los viajeros visitaron zonas de riesgo para malaria y fiebre amarilla, respectivamente. Sólo 4\% de las consultas fueron en el post-viaje. Conclusión: Es posible y fructífero implementar un Centro de Medicina del Viajero en el sector público de salud.

\section{Referencias bibliográficas}

1.- Shaw M. Running a travel clinic. Travel Med Infect Dis 2006; 4:109-26.

2.- http://media.unwto.org/es/pressrelease/2011-09-07/el-turismo-internacionalmuestra-un-saludable-crecimiento-en-laprimera-mit (accedido el 15 de septiembre de 2011).

3.- Acosta R. The pre-travel consultation. En: CDC Health Information for International Travel 2010.The Yellow Book. Mosby Elsevier: 2010; 19-30.

4.- Leggat P. Risk assessment in travel medicine. Travel Med Infect Dis 2006; 4: 127-34.

5.- $\quad$ Spira A. Preparing the traveller. Lancet 2003; 361: $1368-81$.

6.- WHO. Health risks and precautions: general considerations.http:// www.who.int/entity/ith/ ITH 2010 chapter 1 (accedido el 15 de febrero de 2010).

7.- $\quad$ Sleet D, Wallace D, Shlim D. Injuries and safety. En: CDC Health Information for International Travel 2010.The Yellow Book. Mosby Elsevier: 2010: 209-14.

8.- Gushulak B, Mac Pherson W. Globalization of infectious diseases: the impact of migration. Clin Infect Dis 2004; 38: 1742-8.

9.- Wilson M. Population movements and emerging diseases. J Trav Med 1997; 4: 183-6.
10.- Wilder-Smith A, Kee Tai Goh K, Paton N. Experience of Severe Acute Respiratory Syndrome in Singapore: Importation of cases, and defense strategies at the Airport. J Trav Med 2003; 10: 259-62.

11.- Novel Swine-Origin Influenza A (H1N1) Virus Investigation Team. Emergence of a novel swine-origin influenza A (H1N1) virus in humans. N Engl J Med 2009; 360: 2605-15.

12.- http://www.msal.gov.ar/images/stories/ alertas-epidemiologia/2010/alerta-7sarampión-22-8-2010 (accedido el 10 de marzo de 2011).

13.- Hill D, Ericsson C, Pearson R, Keyston J, Freedman D, Kozarsky P, et al. The practice of travel medicine: guidelines by the Infectious Diseases Society of America. Clin Infect Dis 2006; 43: 1499-539.

14.- Freedman D O, Weld L H, Kozarsky P E, Fisk T, Robins R, von Sonnenburg F, et al. Spectrum of disease and relation to place of exposure among ill returned travelers. N Engl J Med 2006; 354 : 119-30.

15.- Leggat P. Sources of health advice given to travellers. J Trav Med 2000; 7: 85-8.

16.- Hatz C, Krause E, Junghanss T H, Grundmann H. Travel advice: a study among swiss and german general practitioners. Selected abstracts from papers presented at $4^{\text {th }}$ International Conference on Travel Medicine, Acapulco, Mexico, 1995. J Trav Med 1995; 2 (2): 114.

17.- Keystone J, Dismukes R, Sawyer L, Kozarsky P. Inadequacies in health recommendations provided for international travelers by North American travel health advisors. J Trav Med 1994; 1: 72-8.

18.- Blamey R. Sobre la prevención de enfermedades infecciosas en viajeros. Rev Chilena Infectol 2011; 28 (1): 64-5.

19.- http://new.paho.org/arg/images/Gallery/ zoonosis/VigilanciaEpizotiasFiebreA.pdf. (accedido el 22 de marzo de 2012).

20.- Wilson M, Weld L, Boggild A, Keystone J, Kain K, von Sonnenburg F, et al. Fever in returned travelers: results from the GeoSentinel Surveillance Network. Clin Infect Dis 2007; 44: 1560-8.

21.- Wilson M and Freedman D. Etiology of travel-related fever. Curr Opin Infect Dis 2007; 20: 449-53.

22.- D'Acremont V, Burnand B, Ambresin A, Genton B. Practice guidelines for evaluation of fever in returning travelers and migrants. J Trav Med 2003; 10 Suppl 2: S25-S00.

23.- Caumes E, Legros F, Duhot D, Cohen J, Arnould P and Mosnier A. Health problems in returning travelers consulting general practitioners. J Trav Med 2008; 15 (6): 457-9. 